\title{
Gamma knife radiosurgery for uveal melanoma ineligible for brachytherapy by the Collaborative Ocular Melanoma Study criteria
}

\author{
Nicola G Ghazi' \\ Christopher S Ketcherside' \\ Jason Sheehan ${ }^{2}$ \\ Brian P Conway' \\ 'Department of Ophthalmology \\ and ${ }^{2}$ Neurosurgery, University \\ of Virginia Health System, \\ Charlottesville, VA, USA
}

Purpose: To report outcomes of Gamma Knife radiosurgery (GKRS) in treating uveal melanoma lesions ineligible for standard brachytherapy.

Methods: A retrospective interventional case series of uveal melanoma patients treated with GKRS between 1996 and 2004 was performed. The main outcome measures were local tumor control, metastasis, and death.

Results: Four patients with uveal melanoma treated with GKS were identified. Three tumors involved the ciliary body and one was macular with its border within $2 \mathrm{~mm}$ of the optic disc. Adequate globe stabilization was achieved by retrobulbar anesthesia in all cases. Pretreatment mean visual acuity was $20 / 30$. Tumor volume as determined by magnetic resonance imaging ranged from 0.05 to $0.30 \mathrm{cc}$. Ultrasonographic greatest tumor diameter and height ranged from 11 to $18 \mathrm{~mm}$ (mean $14.5 \mathrm{~mm}$ ) and 2.9 to $4.5 \mathrm{~mm}$ (mean $3.6 \mathrm{~mm}$ ), respectively. The peripheral dose varied from 16.5 to 30 Gray. Local tumor control was achieved in all cases over a follow up period of 6 to 96 months. Mean final visual acuity was 20/50. One eye was enucleated for neovascular glaucoma and one patient died from liver and lung metastasis.

Conclusions: GKRS for uveal melanoma appears to be safe and effective. The metastasis and mortality rates appear to be comparable to those following brachytherapy and enucleation. Moreover, local tumor control and enucleation rates are similar to those following brachytherapy. The findings in this small series suggest a role for GKRS in the treatment of selected cases of uveal melanomas.

Keywords: gamma knife radiosurgery, radiation therapy, uveal melanoma

\section{Introduction}

Uveal melanoma is the most common primary malignant intraocular tumor in adults. It arises from melanocytes of the uveal tract and most commonly affects the choroid. Choroidal melanoma has a high incidence of metastasis with an overall mortality rate of up to $50 \%$ in 10 years (Olsen et al 2000). Treatment goals include life preservation, prevention of metastasis, and local tumor control with globe/vision preservation. Choroidal melanoma has long been treated with enucleation (Zimmerman et al 1978; Olsen et al 2000). However, it was thought that the procedure may be associated with tumor seeding at the time of surgery after it was reported that the mortality rate increases abruptly during the second year after enucleation (Zimmerman et al 1978). Consequently, other treatment options were explored. Current treatment options include enucleation, eye wall resection, transpupillary thermotherapy, laser photocoagulation, cryotherapy, brachytherapy with various radioisotopes, external beam radiotherapy, and stereotactic radiosurgery including gamma knife surgery (GKS). Studies comparing survival rates following enucleation versus newer treatment modalities, including stereotactic radiosurgery, have suggested similar rates for comparable lesions (Seddon et al 1990; Augsburger et al 1998, 1999; Diener-West et al 2001; Cohen et al 2003). 
We report a small case series of patients treated with GKS for uveal melanomas.

\section{Methods}

In this retrospective interventional case series, 4 patients with uveal melanoma ineligible for brachytherapy by the Collaborative Ocular Melanoma Study (COMS) criteria (COMS 1995; Diener-West et al 2001) were treated with GKS. Patient evaluation included a complete history, preoperative metastatic workup, and a comprehensive ophthalmologic assessment including ocular ultrasonography. A contrast enhanced magnetic resonance imaging (MRI) was part of treatment planning in all cases. Globe immobilization was achieved via a retrobulbar mixture of lidocaine and marcaine in all cases. Attachment of a neurosurgical stereotactic frame to the outer table of the skull was performed under local anesthesia. MRI was then used to calibrate the tumor coordinates. A variable maximal dose was delivered to the tumor such that the $50 \%$ isodose included a $2 \mathrm{~mm}$ margin around the base of the tumor (Table 1). Local tumor response was assessed using serial MRI, ultrasonography, and fundus photography in addition to ophthalmologic examination. Visual outcome, ocular complications and enucleation, metastasis and death rates were also assessed. All data was compiled in a HIPAAcompliant manner.

\section{Results}

Three males and 1 female between the ages of 40 and 76 years (mean 56 years) were treated with GKS. None of the 4 patients was considered a good candidate for brachytherapy (COMS 1995; Diener-West et al 2001). Three tumors involved the ciliary body and one was macular with its nasal border closer than $2 \mathrm{~mm}$ from the optic nerve head. Follow up time ranged from 1 to 8 years. All patients received a single nonfractionated dose of stereotactic radiation except one patient that received 2 successive treatments. The maximum and peripheral doses varied from 40-75 Gray and 16.5-30 Gray, respectively (Table 1 ).
Tables 1 and 2 summarize radiation dose used, treatment outcomes and tumor anatomy as determined by examination, ultrasonography, and MRI. Local tumor control was achieved in all cases (Table 2; Figure1) (Case 4). One patient died 6 years following treatment from liver and lung metastasis. Another patient died due to a hemorrhagic stroke unrelated to the melanoma or treatment. The overall metastasis and tumor-related death rate was $25 \%$.

Two patients maintained 20/20 best corrected Snellen visual acuity while the other two experienced visual deterioration; however, all patients maintained 20/100 or better vision following treatment (Table 2). One patient with a predominantly ciliary body melanoma developed chronic iritis, cataract, and neovascular glaucoma (NVG), and subsequently underwent enucleation 8 years following GKS. Another patient with a choroid and ciliary body melanoma developed serous retinal detachment (SRD) which resolved 4 months later. No other ocular complications were noted.

\section{Discussion}

Gamma knife stereotactic radiosurgery was first envisioned in the 1960's by Lars Leksell, a Swedish neurosurgeon. GKS utilizes a collimating system to deliver a single high-dose radiation with sub-millimeter spatial accuracy and a steep dose gradient. It typically involves only a single treatment although treatment may be repeated on occasion as was the case with one patient in this series.

Previous published series have suggested its efficacy in the treatment of uveal melanomas where the rates of local tumor control, metastasis, and death have ranged from $84 \%$ to $100 \%, 11 \%$ to $26 \%$, and $11 \%$ to $19 \%$, respectively (Marchini et al 1996; Rennie et al 1996; Mullner et al 1998; Vecsei et al 1999; Mueller et al 2000; Woodburn et al 2000; Zehetmayer et al 2000; Haas et al 2002; Simonova et al 2002). Similar rates were observed in this series (Table 3).

Because studies comparing survival rates following enucleation versus newer treatment modalities, including

Table I Tumor location, dimensions and radiation dose

\begin{tabular}{llllll}
\hline Case & Tumor location & MRI tumor volume (cc) & Ultrasound (GLD $\times \mathbf{H ~ m m})$ & Maximum dose (Gy) & Peripheral dose (Gy) \\
\hline $\mathrm{I}$ & Iris/CB & 0.05 & $\mathrm{II} \times 3.6$ & 50 & 30 \\
2 & CB/iris & 0.30 & $17 \times 3.3$ & $66 / 40$ & $20 / 20$ \\
3 & Macular & 0.25 & $12 \times 2.9$ & 50 & 16.5 \\
4 & Choroid/CB & 0.22 & $18 \times 4.5$ & 75 & 30 \\
\hline
\end{tabular}

Abbreviations: CB, ciliary body; MRI, magnetic resonance imaging; cc, cubic centimeter; GLD, greatest linear dimension; H, height; mm, millimeter; Gy, Gray. 
Table 2 Treatment outcomes

\begin{tabular}{|c|c|c|c|c|c|c|c|c|}
\hline case & MRI vol i (cc) & MRI vol f (cc) & US GLD $\times \mathbf{H}(\mathrm{mm}) \mathbf{i}$ & US GLD $\times \mathbf{H}(\mathbf{m m}) \mathbf{f}$ & VA $i$ & VA f & Mets & Death \\
\hline $\mathrm{I}$ & 0.05 & 0.03 & $\mathrm{II} \times 3.6$ & $11 \times 3.6$ & $20 / 20$ & $20 / 20$ & No & No \\
\hline 2 & 0.30 & 0.25 & $17 \times 3.3$ & $17 \times 3.3$ & $20 / 20$ & $20 / 60$ & No & No \\
\hline 3 & 0.25 & 0.25 & $12 \times 2.9$ & $12 \times 3.0$ & $20 / 50$ & $20 / 100$ & No & Yes \\
\hline 4 & 0.22 & $(0.22)$ & $18 \times 4.5$ & $18 \times 2.7$ & $20 / 20$ & $20 / 20$ & Yes & Yes \\
\hline
\end{tabular}

Abbreviations: MRI, magnetic resonance imaging; vol, volume; I, initial; cc, cubic centimeter; $f$, final; $U S$, ultrasound; GLD, greatest linear dimension; $\mathrm{H}$, height; mm, millimeter; VA, best corrected Snellen visual acuity; Mets, metastasis.

stereotactic radiosurgery, suggested similar rates for comparable lesions (Seddon et al 1990; Augsburger et al 1998, 1999; Diener-West et al 2001; Cohen et al 2003), and because reported local tumor control rate following GKS appear comparable to that following brachytherapy (Marchini et al 1996; Rennie et al 1996; Mullner et al 1998; Vecsei et al 1999; Mueller et al 2000; Woodburn et al 2000; Zehetmayer et al 2000; Haas et al 2002; Simonova et al 2002), we offer GKS to patients who would otherwise require enucleation or unconventional brachytherapy. This includes those with choroidal melanomas that do not meet the COMS eligibility criteria for brachytherapy
(COMS 1995; Diener-West et al 2001) or large iris and/or ciliary body melanomas.

GKS may be advantageous because it is an outpatient procedure, requires only local anesthesia, offers a steep dose gradient where only low radiation doses affect surrounding ocular tissue, and does not require ocular manipulation. An advantage over brachytherapy is that no subsequent procedure for plaque removal is necessary.

Some of the severe complications that may be associated with GKS include NVG, radiation retinopathy, and the need for enucleation. In one study these complications occurred at a rate of up to $47 \%, 84 \%$, and $22 \%$, respectively over

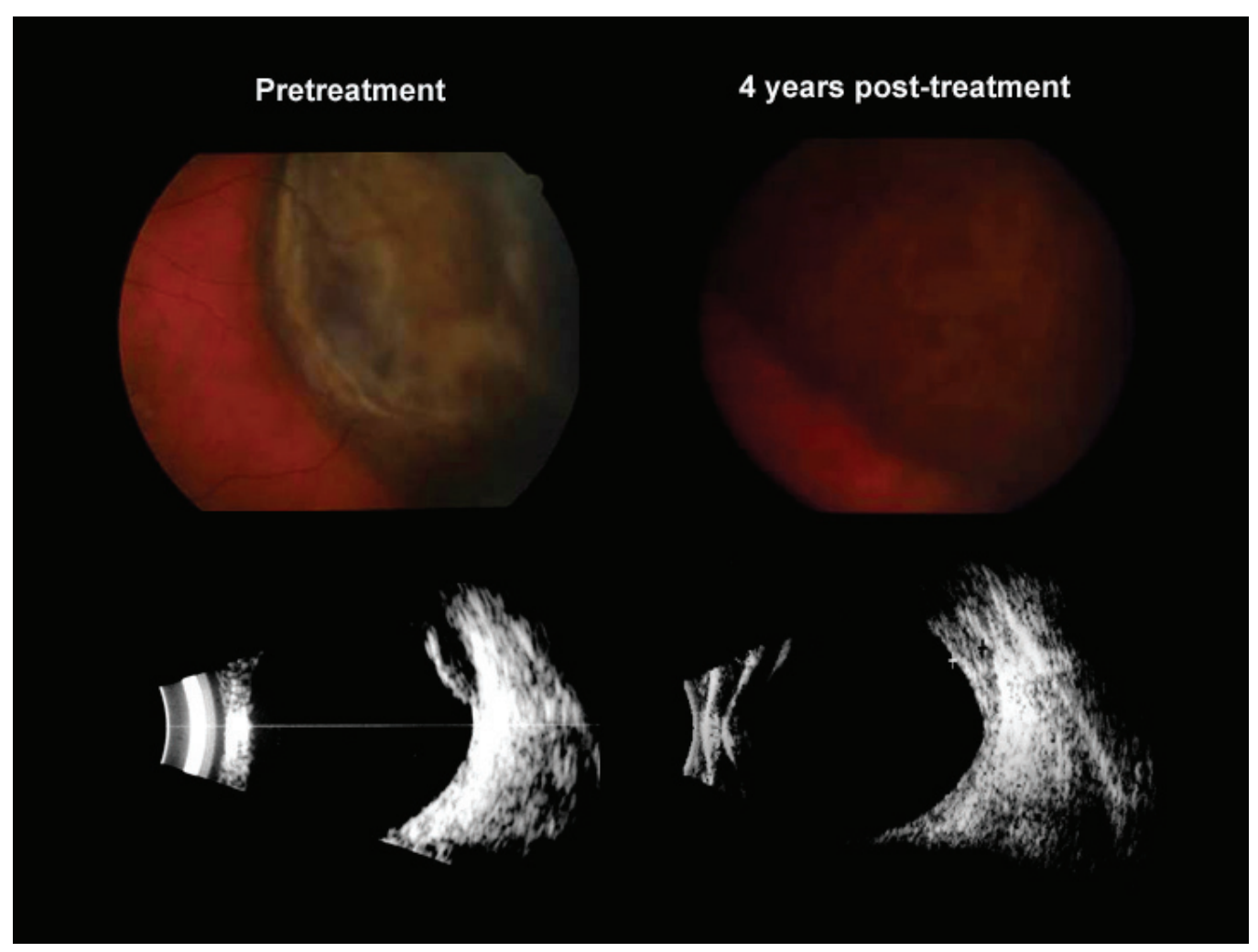

Figure I Appearance of a choroidal/ciliary body melanoma of a 53-year-old male before (left) and after (right) gamma knife radiosurgery. Note change in tumor texture on fundus photography and thickness reduction from 4.5 to $2.7 \mathrm{~mm}$ by ultrasonography 4 years after treatment. 
Table 3 Literature review

\begin{tabular}{|c|c|c|c|c|c|}
\hline Study & $\mathbf{n}$ & F/U (yrs) & Local control rate (\%) & Metastasis rate (\%) & Death rate (\%) \\
\hline Cohen et al 2003 & 78 & $U_{p}$ to 10 & NR & 26 & NR \\
\hline Simonova et al 2002 & 81 & Up to 5 & 84 & 19 & 19 \\
\hline Woodburn et al 2000 & 11 & $<1.5$ & 100 & NR & NR \\
\hline Mueller et al 2000 & 35 & $>1$ & 97 & NR & NR \\
\hline Zehetmayer et al 2000 & 62 & $\mathrm{I}-4.5$ & 98 & 11 & 10 \\
\hline Marchini et al 1996 & 12 & $<1$ & 100 & NR & NR \\
\hline Vecsei et al 1999 & 9 & 2 & 100 & II & 11 \\
\hline Current study & 4 & $\mathrm{I}-8$ & 100 & 25 & 25 \\
\hline
\end{tabular}

Abbreviations: n, number of patients in study; F/U, follow up; yrs, years; <, less than; \%, percentage; NA, not reported.

7 years following a single high marginal dose (40-80 Gy) (Haas et al 2002). The incidence of such complications increases with tumors greater than $8 \mathrm{~mm}$ in height, ciliary body tumors, macular tumors, older patients, higher radiation dose, and higher target volume (Zehetmayer et al 2000). Other complications include dry eye, keratopathy, uveitis, cataract, optic neuropathy, SRD, and vitreous hemorrhage. In this series, 1 patient developed NVG and required enucleation while another developed SRD that resolved spontaneously.

To date there has been no multi-center trial to assess dosimetry, safety and efficacy of GKS. However, data from several case series suggest that GKS has comparable local tumor control rate, metastasis rate, mortality rate and complication rate to brachytherapy (Seddon et al 1990; Marchini et al 1996; Rennie et al 1996; Augsburger et al 1998, 1999; Mullner et al 1998; Vecsei et al 1999; Mueller et al 2000; Woodburn et al 2000; Zehetmayer et al 2000; Diener-West et al 2001; Haas et al 2002; Simonova et al 2002; Cohen et al 2003), as well as similar metastasis and mortality rates to enucleation, suggesting that tumor behavior may be more dependent upon intrinsic tumor characteristics, such as its cytogenetics (Baggetto et al 2005). Our findings, like previous studies, suggest that GKS may be an appropriate alternative for treating uveal melanoma, especially when lesions are ineligible for conventional brachytherapy.

\section{Disclosure}

The authors report no conflicts of interest in this work.

\section{References}

Augsburger JJ, Correa ZM, Freire J, et al. 1998. Long-term survival in choroidal and ciliary body melanoma after enucleation versus plaque radiation therapy. Ophthalmology, 105:1670-8.

Augsberger JJ, Schneider S, Freire J, et al. 1999. Survival following enucleation versus plaque radiotherapy in statistically matched subgroups of patients with choroidal melanomas: results in patients treated between 1980 and 1987. Graefes Arch Clin Exp Ophthalmol, 237:558-67.
Baggetto LG, Gambrelle J, Dayan G, et al. 2005. Major cytogenetic aberrations and typical multidrug resistance phenotype of uveal melanoma: current views and new therapeutic prospects. Cancer Treat Rev, 31:361-79.

Cohen VM, Carter MJ, Kemeny A, et al. 2003. Metastasis-free survival following treatment for uveal melanoma with either stereotactic radiosurgery or enucleation. Acta Ophthalmol Scand, 81:383-8.

[COMS] Collaborative Ocular Melanoma Study Group. 1995. COMS Manual of Procedures. Springfield, Va: National Technical Information Service. Accession No. PB95-179693.

Diener-West M, Reynolds SM, Agugliaro DJ, et al. 2001. The COMS randomized trial of iodine 125 brachytherapy for choroidal melanoma, III: initial mortality findings. COMS Report No. 18. Arch Ophthalmol, 119:969-82.

Haas A, Pinter O, Papaefthymiou G, et al. 2002. Incidence of radiation retinopathy after high-dosage single-fraction gamma knife radiosurgery for choroidal melanoma. Ophthalmology, 109:909-13.

Marchini G, Gerosa M, Piovan E, et al. 1996. Gamma Knife stereotactic radiosurgery for uveal melanoma: clinical results after 2 years. Stereotact Funct Neurosurg, 66(Suppl 1):208-13.

Mueller AJ, Talies S, Schaller UC, et al. 2000. Stereotactic radiosurgery of large uveal melanomas with the gamma-knife. Ophthalmology, 107:1381-7.

Mullner K, Langmann G, Pendl G, et al. 1998. Echographic findings in uveal melanomas treated with the Leksell gamma knife. $\mathrm{Br} J$ Ophthalmol, $82: 154-8$.

Olsen KR, Murray TG, Johnson RN. 2000. Enucleation and plaque treatment. In: Albert DM, Jakobiec FA, Azar DT, et al. (eds). Principles and Practice of Ophthalmology. Philadelphia: Saunders Company, pp. 5028-46.

Rennie I, Forster D, Kemeny A, et al. 1996. The use of single fraction Leksell stereotactic radiosurgery in the treatment of uveal melanoma. Acta Ophthalmol Scand, 74:558-62.

Seddon JM, Gragoudas ES, Egan KM, et al. 1990. Relative survival rates after alternative therapies for uveal melanoma. Ophthalmology, 97:769-77.

Simonova G, Novotny J, Jr, Liscak R, et al. 2002. Leksell gamma knife treatment of uveal melanoma. J Neurosurg, 97:635-9.

Vecsei PV, Kircher K, Nagel G, et al. 1999. Ocular arterial blood flow of choroidal melanoma eyes before and after stereotactic radiotherapy using Leksell gamma knife: 2 year follow up. Br J Ophthalmol, 83:1324-8.

Woodburn R, Danis R, Timmerman R, et al. 2000. Preliminary experience in the treatment of choroidal melanoma with gamma knife radiosurgery. J Neurosurg, 93(Suppl 3):177-9.

Zehetmayer M, Kitz K, Menapace R, et al. 2000. Local tumor control and morbidity after one to three fractions of stereotactic external beam irradiation for uveal melanoma. Radiother Oncol, 55:135-44.

Zimmerman LE, McLean IW, Foster WD. 1978. Does enucleation of the eye containing a malignant melanoma prevent or accelerate the dissemination of tumour cells. Br J Ophthalmol, 62:420-5. 